\title{
Photoinduced magneto-optic Kerr effects in asymmetric semiconductor microcavities
}

\author{
D. Pereda Cubian, ${ }^{1,2}$ M. Haddad, ${ }^{3}$ R. André,${ }^{4}$ R. Frey, ${ }^{1}$ G. Roosen, ${ }^{1}$ J. L. Arce Diego, ${ }^{2}$ and C. Flytzanis ${ }^{5}$ \\ ${ }^{1}$ Laboratoire Charles Fabry de l'Institut d'Optique (CNRS UMR 8501), Université Paris Sud, Bât. 503, F-91403 Orsay Cédex, France \\ ${ }^{2}$ Departamento de Tecnologia Electronica e Ingenieria de Sistemas y Automatica, Universidad de Cantabria, \\ E-39005 Santander, Espania \\ ${ }^{3}$ Laboratoire d'Optique Quantique, Ecole Polytechnique and CNRS, F-91128 Palaiseau Cédex, France \\ ${ }^{4}$ Laboratoire de Spectrométrie Physique (CNRS UMR 558), Université J. Fourier-Grenoble, \\ B.P. 87, F-38402 Saint Martin d'Hères Cédex, France \\ ${ }^{5}$ Laboratoire de Physique de la Matière Condensée, Ecole Normale Supérieure and CNRS, \\ 24 Rue L'Homond, F-75231 Paris Cédex, France
}

(Received 24 May 2002; revised manuscript received 12 September 2002; published 14 January 2003)

\begin{abstract}
Giant photoinduced magneto-optic Kerr effects are predicted and measured in asymmetric semiconductor microcavities with a totally reflecting rear mirror operated in the limit of the strong coupling regime. The microcavity is modeled by two coupled Fabry-Perot cavities and use is made of the optical scattering matrices to derive its characteristics. The giant photoinduced rotations and phase changes are traced to the saturation, blueshift, and pseudo-Zeeman splitting of the exciton transition. Modeling the lower and upper polariton transitions by two different two-level systems qualitatively accounts for the main spectral features: the photoinduced Kerr rotations and phase changes are due to the modifications of the coupling existing between the cavity and exciton modes, due to the photoinduced changes of the exciton characteristics. The influence of spin relaxation on the rotation and ellipticity spectra is also analyzed; it confirms the gyrotropic nature of the interaction which depends strongly on the difference between the densities of counter-rotating circularly polarized excitons and only weakly on their sum as is the case in previous isotropic studies. Measurements of photoinduced Kerr rotations performed at a temperature of $50 \mathrm{~K}$ in a microcavity containing a single semimagnetic semiconductor quantum well confirm the effectiveness of the effect with polarization rotations of $10^{\circ}$ around the lower polariton frequency at a pump fluence of only $2 \mu \mathrm{J} / \mathrm{cm}^{2}$.
\end{abstract}

DOI: 10.1103/PhysRevB.67.045308

PACS number(s): 78.67.De, 42.65.-k

\section{INTRODUCTION}

The study of exciton-polariton modes received a renewed attention in the recent years with the use of quantum microcavities where the material (exciton) and electromagnetic (photon) components can be independently and artificially modified through appropriate tailoring of microcavities and quantum well characteristics. ${ }^{1,2}$ The coupling of the exciton and cavity modes in these so-called cavity polariton modes can be easily modified by static electric ${ }^{2,3}$ and magnetic ${ }^{4-9}$ fields. The modification of the cavity-polariton characteristics by intense laser pulses has also been investigated and revealed polariton bleaching and many-body interaction effects. ${ }^{10}$ In these studies only the isotropic (scalar) part of these interactions could be assessed since the gyrotropic part is canceled out in the chosen configurations.

Here we assess the gyrotropic part of these photoinduced changes through an experimental and theoretical study of the polarization effects occurring at high exciton densities in a microcavity operated in the strong coupling regime. In this nonlinear process, a circularly polarized pump pulse induces polarization changes on a linearly polarized incident probe beam due to the huge polarization dependent modifications of the light induced cavity-exciton coupling. In Sec. II we schematically present the experimental configuration used in our study. In Sec. III we present the setup used in our experiment and show that polarization rotations of a weak probe beam as large as $10^{\circ}$ can be photoinduced by $2 \mu \mathrm{J} / \mathrm{cm}^{2}$ fluence circularly polarized pump pulses. In Sec. IV we de- scribe the principle of the calculations based on optical scattering matrices which allow the determination of the magneto-optic Kerr rotation and phase change photoinduced by a circularly polarized pump pulse. In Sec. V we apply the model to the case of resonant optical nonlinearities resulting from the photogeneration of polarized excitons. The nonlinearities considered in this section, i.e., saturation, blueshift, and pseudo-Zeeman splitting of the exciton transition induced by circularly polarized pump pulses are considered decoupled from population and spin relaxation processes. The corresponding photoinduced polarization rotation and ellipticity spectra are compared and their physical interpretation given in terms of the shift of the polariton frequencies due to the modifications of the exciton transition characteristics. The role played by spin relaxation processes on the photoinduced Kerr rotations and phase changes is analyzed in Sec. VI in two particular cases: for synchroneous pump and probe pulses when the exciton spin relaxation time is of the order of the pulse durations and for delayed short duration probe pulses in the case of long-lived exciton spins.

\section{EXPERIMENTAL CONFIGURATION}

The device considered in this study is an asymmetric microcavity operated in the strong coupling regime (see Fig. 1). A circularly polarized short duration pump pulse is sent onto the device at some small angle in order to generate polarized excitons. The frequency of the pump pulse is resonant with one of the polariton modes in order to instantaneously gen- 


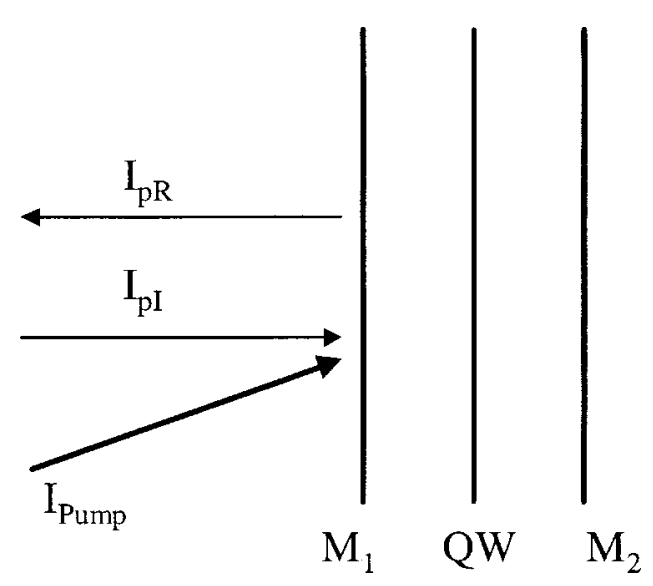

FIG. 1. Schematic view of the microcavity.

erate the polarized excitons. A linearly polarized incident probe beam of wavelength $\lambda$ is sent perpendicularly to the microcavity. The polarization state of the probe beam reflected by the device is analyzed, considering both the rotation angle and the ellipticity of the beam polarization.

The microcavity is closed by a rear mirror $M_{2}$ which is nearly totally reflecting and a partially reflecting front mirror $M_{1}$. The asymmetric cavity configuration is chosen since such a device operated in reflection allows the highest sensitivities provided the reflectivity of the front mirror is adjusted for optimizing the desired effect. It is the case, for instance, for intracavity absorption, ${ }^{11}$ phase shift, ${ }^{12}$ Bragg diffraction, ${ }^{13}$ or Kerr polarization rotation. ${ }^{14}$

For the sake of simplicity we consider here the simplest case of a microcavity with a single quantum well (QW) located at the middle of a $\lambda / 2 n$-thick transparent intracavity material of refractive index $n$. Due to the strong absorption occurring at the resonance frequency of the quantum well exciton, the thin quantum well acts as an intermediate mirror exhibiting a very small reflectivity bandwith. The strong coupling regime can therefore be viewed as resulting of the coupling of three Fabry-Perot cavities (the cavity $M_{1}-M_{2}$ of thickness $l$ and the two cavities $M_{1}-\mathrm{QW}$ and QW- $M_{2}$ of thickness $l / 2$ ). In this study we consider the simplest case of a tuned cavity when the cavity resonance frequency $\omega_{c}$ is made equal to the exciton resonance frequency $\omega_{0}$. This situation corresponds to the maximum coupling between the exciton and cavity modes with polaritons exhibiting a fully mixed exciton-cavity character.

In the case of Kerr polarization effects, the cavity is optimized for an operation of the microcavity in the limit of the strong coupling regime when the Rabi splitting $2 \Omega_{0}$ is nearly equal to the polariton linewidth. Indeed, as magneto-optic Kerr effects have a gyrotropic nature, they result from the difference between contributions due to the counter-rotating polarizations. In this way, only a small polarizationdependent variation of the exciton resonance frequency or a slight saturation of the exciton transition can produce a huge modification of the phase of the beam reflected by the microcavity, thus providing a high sensitivity for this polarization device. This behavior was already observed in Kerr rotation measurements with increases in sensitivity of several

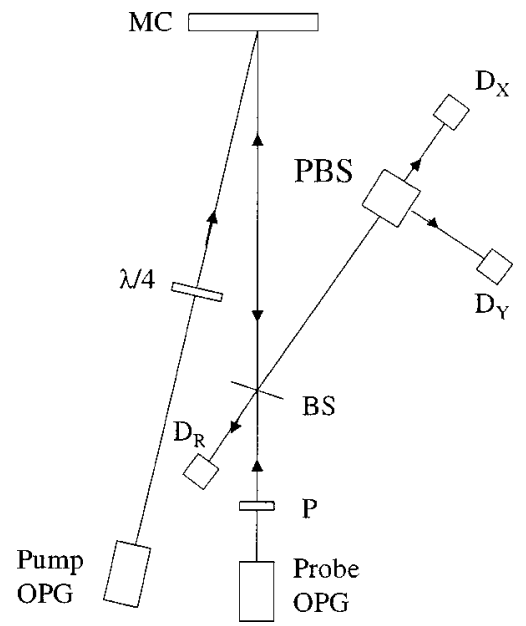

FIG. 2. Experimental setup. MC: microcavity, OPG: optical parametric generator, $\lambda / 4$ :- quarter wave plate, $P$ : polarizer, BS: beam splitter, PBS: polarization beam splitter, $D_{R}, D_{X}, D_{Y}$ : detectors.

orders of magnitudes for the Verdet constant ${ }^{14}$ when compared to the case of symmetrical cavities. ${ }^{15}$

\section{EXPERIMENTAL RESULTS}

Experiments were conducted at relatively low temperature $(T=50 \mathrm{~K})$ using a microcavity placed in a cryostat. This sample was grown by molecular beam epitaxy on a $\mathrm{Cd}_{0.88} \mathrm{Zn}_{0.12} \mathrm{Te}$ [100] oriented substrate. The back and front cavity mirrors, of respective reflection coefficients $R_{2}$ $=0.96$ and $R_{1}=0.76$, were formed by stacking $\lambda / 4$-thick $\mathrm{Cd}_{0.4} \mathrm{Mg}_{0.6} \mathrm{Te} / \mathrm{Cd}_{0.75} \mathrm{Mn}_{0.25} \mathrm{Te}$ pairs. A 70- $\AA$-thick $\mathrm{Cd}_{0.95} \mathrm{Mn}_{0.05} \mathrm{Te}$ quantum well was grown in the middle of the $\lambda / 2$-thick $\mathrm{Cd}_{0.4} \mathrm{Mg}_{0.6} \mathrm{Te}$ intracavity material. This configuration of the asymmetric microcavity operated in the limit of the strong coupling regime (for a cavity-exciton coupling strength only slightly larger than the polariton linewidth) was chosen in order to maximize magneto-optic Kerr rotation angles in the presence of small amplitude magnetic fields. ${ }^{14}$ Note that due to the high energy of the band gap of the semiconductor materials used for the Bragg mirrors, no significant gyrotropic effect is expected for the cavity mirrors, so that the polarization modifications occur only owing to the microcavity.

The experimental setup used for the study of this microcavity is shown in Fig. 2. Two independently tunable laser sources are used: the probe and pump beams which are arising from two identical optical parametric generators (OPG's) pumped by a picosecond duration frequency-tripled Nd-YAG laser. ${ }^{16,17}$ The pump source is used to generate circularly polarized excitons. The probe source is used for recording the reflectivity and polarization rotation spectra. Indeed, as the Kerr rotations and phase changes measured in this experiment are low, phase measurements are impossible due to the small undesirable and uncompensable dephasing brought by the cryostat windows. The pump and probe beams are focused at slightly tilted and normal incidences onto the microcavity (MC), respectively. The beam splitter (BS) provides 
an energy reference of the probe pulse and also allows for the extraction of the probe beam reflected by the microcavity. This reflected beam is separated into two orthogonally polarized beams by the polarizing beam splitter (PBS). The linear polarization of the incident beam is set at $45^{\circ}$ of the $X$ and $Y$ directions of the PBS. With no pump pulse, the $X$ and $Y$ intensities are equal. In the presence of the circularly polarized pump pulse the polarization direction of the probe beam is rotated by an angle $\theta$ and dephased by an angle $\varphi$ by the microcavity. The intensities $I_{X}$ and $I_{Y}$ measured on $X$ and $Y$ polarization states, respectively, are then given by

$$
\begin{aligned}
& I_{X}=I_{0}\left[\cos ^{2} \varphi \cos ^{2}\left(\frac{\pi}{4}+\theta\right)+\sin ^{2} \varphi \sin ^{2}\left(\frac{\pi}{4}+\theta\right)\right], \\
& I_{X}=I_{0}\left[\cos ^{2} \varphi \sin ^{2}\left(\frac{\pi}{4}+\theta\right)+\sin ^{2} \varphi \cos ^{2}\left(\frac{\pi}{4}+\theta\right)\right],
\end{aligned}
$$

where $I_{0}=I_{X}+I_{Y}$ is the reflected intensity. For the small values of $\varphi$ reached in our experiment, the reflectivity $R$ of the microcavity and $\theta$ are calculated using the formulas

$$
\begin{gathered}
R=\frac{I_{X}+I_{Y}}{I_{R}}, \\
\theta=\frac{1}{2} \operatorname{Arcsin}\left[\frac{I_{Y}-I_{X}}{I_{X}+I_{Y}}\right],
\end{gathered}
$$

where $I_{R}$ is the incident intensity.

Note that no polarization rotation occurs in the linear regime without pump pulses. When a circularly polarized high fluence pump pulse is sent onto the microcavity together with the probe pulse a photoinduced polarization rotation of the linearly polarized probe pulse is observed, and reversing the circular polarization of the pump pulse only changes the sign of the polarization rotation. Figures 3(a) and 3(b) show the polarization rotation spectrum photoinduced by a $\hat{\sigma}_{-}$circularly polarized pump pulse and the reflectivity spectra recorded with and without the pump pulse present (solid squares and open circles, respectively). In this experiment the pump fluence was $F=2 \mu \mathrm{J} / \mathrm{cm}^{2}$ and the frequency of the pump beam chosen to selectively excite the lower polariton mode (at a pump photon energy $E_{p}=1.708 \mathrm{eV}$ ).

The photoinduced rotation spectrum shown in Fig. 3(a) exhibits a large negative rotation peak (about $-10^{\circ}$ ) around the lower polariton mode and almost no rotation occurs around the upper polariton mode. In the presence of the pump pulse the reflectivity spectrum is blueshifted by about $0.3 \mathrm{meV}$ and the exciton transition is slightly saturated as attested by the narrowing of this reflectivity spectrum [see open circles in Fig. 3(b)]. The straight line in Fig. 3(a) shows the best fit to the experimental photoinduced rotation spectrum obtained using the model presented in Sec. IV. The agreement between experiment and theory is rather good and the calculated parameters $(0.28 \pm 0.02 \mathrm{meV}$ and $1.5 \pm 0.1 \%$ for the blueshift and saturation of the exciton transition, respectively) that give the best fit are in good agreement with the values extracted from the reflectivity measurements $(0.3$

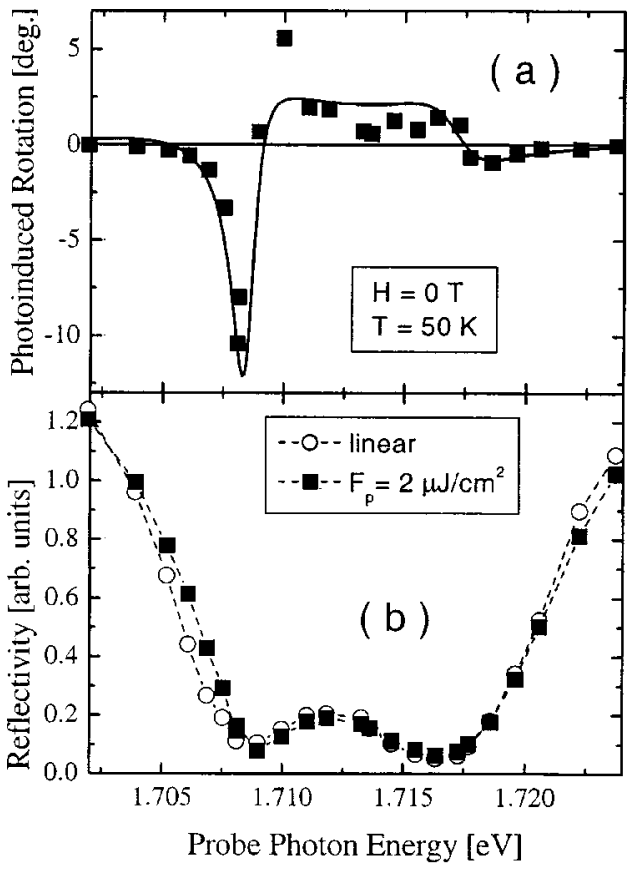

FIG. 3. Kerr rotation spectrum photoinduced by a $2 \mu \mathrm{J} / \mathrm{cm}^{2}$ fluence $\sigma_{-}$polarized pump pulse (a) and reflectivity spectra recorded with and without the pump pulse (b). The continuous line in (a) is the best fit obtained using the model of Sec. III and the dashed lines in (b) are only guides for the eyes.

meV for the blueshift of the exciton transition and a small saturation which could not be measured precisely).

We also point out that the photoinduced polarization rotation spectrum was recorded at a temperature of $50 \mathrm{~K}$. The large value obtained for the measured photoinduced rotations at this relatively high temperature strongly indicates that large photoinduced polarization rotations can be obtained at room temperature using a microcavity specially designed to work in the strong coupling regime at this temperature.

\section{MODEL}

The Kerr polarization rotation $\theta$ and ellipticity or phase change $\varphi$ experienced by the linearly polarized incident probe beam after its reflection by the microcavity are calculated using the expressions

$$
\begin{gathered}
\theta=\frac{1}{2}\left(\operatorname{Arg}\left[R_{-}\right]-\operatorname{Arg}\left[R_{+}\right]\right), \\
\varphi=\operatorname{Arctan}\left[\frac{\left|R_{+}\right|-\left|R_{-}\right|}{\left|R_{+}\right|+\left|R_{-}\right|}\right],
\end{gathered}
$$

where $R_{ \pm}=A_{R_{ \pm}} / A_{I \pm}$ are the amplitude reflectivities of the microcavity for the counter-rotating circular polarizations $\hat{\sigma}_{ \pm}\left(A_{R_{ \pm}}\right.$and $A_{I \pm}$ are the reflected and incident amplitudes of the probe beam for the $\hat{\sigma}_{ \pm}$polarizations, respectively). The circularly polarized amplitude reflectivities $R_{ \pm}$are obtained using the optical scattering matrices. ${ }^{18}$ 
For the calculation of $R_{ \pm}$the only parameters concern the Bragg mirrors and the quantum well reflector. The Bragg mirrors exhibit reflection coefficients given by

$$
r_{1,2}=\sqrt{R_{1,2}} \exp \left(-i \Phi_{1,2}\right),
$$

where $R_{1,2}$ and $\Phi_{1,2}=B_{1,2} \cdot\left(\omega-\omega_{1,2}\right)$ are the intensity reflection coefficients and the frequency-dependant phase shifts experienced by the reflected beam respectively, with $\omega_{1,2}$ and $B_{1,2}$ the central frequencies and phase slopes, respectively. ${ }^{19}$ For the sake of simplicity the same central frequency $\omega_{M}$ $=\omega_{1}=\omega_{2}=\omega_{C}$ is used all along this study. This choice also corresponds to the experimental situation described in Sec. III. The quantum well reflector exhibits circularly polarized reflection and transmission coefficients given by ${ }^{19}$

$$
\begin{gathered}
r_{ \pm}=i \frac{\Gamma_{0 \pm}}{\omega-\omega_{0 \pm}-i\left(\gamma_{ \pm}+\Gamma_{0 \pm}\right)}, \\
t_{ \pm}=\left(1+r_{ \pm}\right) \exp \left(-i \Phi_{\mathrm{QW}}\right) .
\end{gathered}
$$

In Eqs. 6(a) and 6(b) $\omega_{0 \pm}, \Gamma_{0 \pm}$, and $\gamma_{ \pm}$are the resonance frequencies, oscillator strengths, and linewidths, respectively, for the $\hat{\sigma}_{ \pm}$polarizations, and $\Phi_{\mathrm{OW}}\left(\Phi_{\mathrm{QW}}=2 \pi n e / \lambda\right.$, with $e$ the quantum well thickness) is the non resonant phase shift introduced by the quantum well. With no pump pulse the polarization dependent parameters reduce to the linear values $\omega_{0}, \Gamma_{0}$, and $\gamma$, which only depend on the quantum well characteristics.

All the parameters of the microcavity considered in our study are given using the homogenous linewidth $\gamma$ of the exciton transition as the frequency unit. Indeed, in the next sections the Kerr rotations and phase changes are studied as a function of the normalized frequency detuning $(\omega$ $\left.-\omega_{0}\right) / \gamma$. For the calculations of Secs. V and VI we use $R_{2}$ $=1$ and $B_{2} \gamma=0.104$ and $R_{1}=0.82$ and $B_{1} \gamma=0.072$ for mirrors $M_{1}$ and $M_{2}$, respectively, and $\Gamma_{0} / \gamma=0.06$ and $\Phi_{\mathrm{QW}}$ $=0.175$ for the quantum well characteristics. These values are typical for II-VI semimagnetic semiconductor microcavities, but our analysis can be applied to any microcavity.

\section{DIFFERENT NONLINEARITIES}

When a circularly polarized pump pulse is sent onto the microcavity, the resonance frequency, oscillator strength, and linewidth of the quantum well can be modified through several physical mechanisms. Here, we discuss the cases of the saturation of the quantum well exciton due to band filling effects and many body interactions, ${ }^{20,21}$ that of the many body interaction induced blueshift of the exciton transition ${ }^{21}$ and that of the pseudo-Zeeman effect induced by the magnetization which can be created in the quantum well by spin transfer when the intracavity or (and) the quantum well is (are) magnetic. ${ }^{22}$ For the sake of simplicity and clarity, in this section we consider these photoinduced interactions before population and spin relaxation processes set in for a modification of the quantum well properties induced by $\hat{\sigma}_{+}$ polarized pump pulses.

In the strong coupling regime of the microcavity, the lower and upper polariton frequencies $\omega_{L}$ and $\omega_{U}$ and line- widths $\gamma_{L}$ and $\gamma_{U}$ are given by the following expressions derived from Ref. 23:

$$
\begin{aligned}
\omega_{L, U}+i \gamma_{L, U}= & \left(\omega_{0}+\omega_{C}\right) / 2+i\left(\gamma_{0}+\gamma_{C}\right) / 2 \\
& \mp \sqrt{V_{0}^{2}+\frac{1}{4}\left[\omega_{0}-\omega_{C}-i\left(\gamma_{C}-\gamma_{0}\right)\right]^{2}}
\end{aligned}
$$

with

$$
\begin{gathered}
V_{0}^{2}=\frac{\left(\sqrt{R_{1}}+\sqrt{R_{2}}+2 \sqrt{R_{1} R_{2}}\right) \Gamma_{0}}{\left(B_{1}+B_{2}+2 n l / c\right) \sqrt{R_{1} R_{2}}}, \\
\gamma_{C}=\frac{1-\sqrt{R_{1} R_{2}}}{\left(B_{1}+B_{2}+2 n l / c\right) \sqrt{R_{1} R_{2}}},
\end{gathered}
$$

where $V_{0}$ and $\gamma_{C}$ represent the pure coupling constant between the cavity and exciton modes and the halfwidth of the cavity mode, respectively. Using the values given in Sec. IV, we get $V_{0} / \gamma_{0}=1.15$ and $\gamma_{C} / \gamma_{0}=0.56$ and the Rabi splitting $\Omega=\left(\omega_{U}-\omega_{L}\right) / 2$ is slightly smaller than $V_{0}\left(\Omega / \gamma_{0}=1.13\right)$.

As $V_{0}$ and $\omega_{0}$ are modified at high laser fluences, the lower and upper polariton frequencies are modified in the presence of a $\hat{\sigma}_{+}$polarized pump pulse. This effects occur irrespective of the physical origin of the nonlinearity. If we consider the device as a set of double two-level systems of respective resonance frequencies $\omega_{L}$ and $\omega_{U}$, the Kerr polarization rotation $\theta_{L, U}$ and phase change $\varphi_{L, U}$ can be evaluated around each resonance using the simple expressions

$$
\begin{gathered}
\theta_{L, U}=\frac{\omega L}{2 c}\left(n_{L, U_{-}}^{\prime}-n_{L, U_{+}}^{\prime}\right), \\
\varphi_{L, U}=\operatorname{Arctg}\left[\tanh \left[\frac{\omega L}{2 c}\left(n_{L, U_{-}}^{\prime \prime}-n_{L, U_{+}}^{\prime \prime}\right)\right]\right]
\end{gathered}
$$

with $L$ the equivalent length of the device and where

$$
n_{L, U_{ \pm}}=n_{L, U_{ \pm}}^{\prime}+i n_{L, U_{ \pm}}^{\prime \prime}=n_{0}+A \frac{\left(\omega_{L, U_{ \pm}}-\omega-i \Gamma_{L, U_{ \pm}}\right)}{\left[\left(\omega_{L, U_{ \pm}}-\omega\right)^{2}+\Gamma_{L, U_{ \pm}}^{2}\right]}
$$

are the complex refractive indices for the $\hat{\sigma}_{ \pm}$polarizations around the polariton resonance frequencies $\omega_{L, U_{ \pm}}, A$ being a constant related to the oscillator strength of the two-level system. In Eq. (11) the polarization dependence of $A$ is not considered since, for the physical mechanisms responsible for the photoinduced Kerr effects considered hereafter, the amplitude suppression is much less effective than the resonance frequency shift for the polarization rotation and phase changes. As a consequence, for small $\hat{\sigma}_{ \pm}$variations of the polariton frequencies, the polarization rotation spectra exhibit an "absorption-type" shape centered on $\omega_{L, U}$ with a maximum rotation angle proportional to $\Delta \omega_{L, U}$ $=\operatorname{Re}\left(\omega_{L, U_{+}}-\omega_{L, U_{-}}\right)$given by

$$
\Delta \omega_{L, U}=\frac{1}{2}\left(\delta \omega_{0_{+}}-\delta \omega_{0_{-}}\right) \pm\left(\Delta \Omega_{+}-\Delta \Omega_{-}\right),
$$




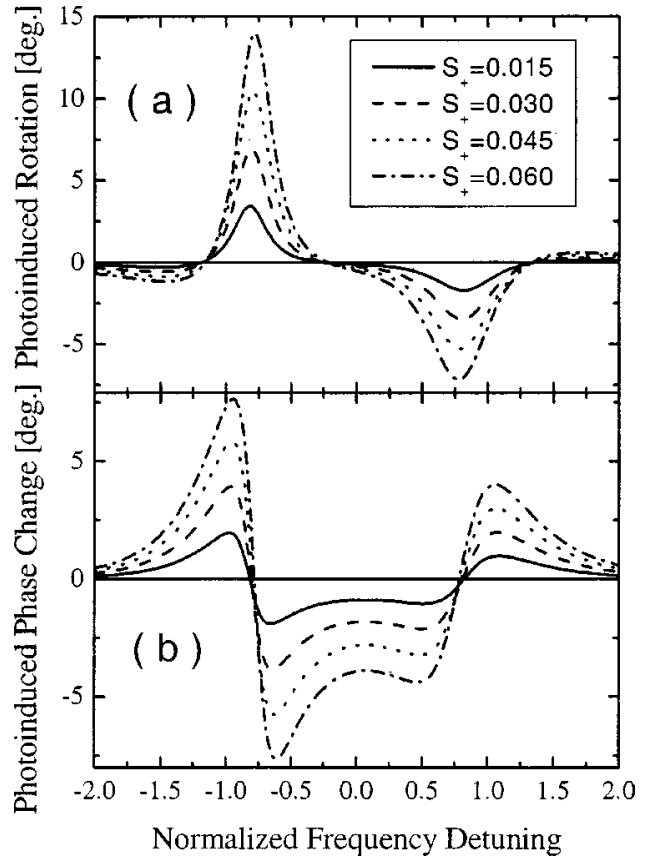

FIG. 4. Photoinduced Kerr rotation (a) and phase change (b) spectra for various saturations of the $\sigma_{+}$polarized exciton transition.

where $\delta \omega_{0_{ \pm}}=\omega_{0_{ \pm}}-\omega_{0}$ are the frequency changes of the exciton transition and $\Delta \Omega_{ \pm}=\Omega_{ \pm}-\Omega$ are the variations of the Rabi frequency $\Omega$ for the $\hat{\sigma}_{ \pm}$circular polarization, respectively. In the same manner, the ellipticity spectra exhibit a dispersion-type shape centered on $\omega_{L, U}$ with a maximum phase change angle proportional to $\Delta \omega_{L, U}$.

Let us remark that the polariton frequencies and linewidths given by Eq. (7) are not obtained from the reflectivity of the microcavity ${ }^{23}$ so that quantitative values cannot be derived from Eq. (10) for the photoinduced polarization rotation and phase change. However, as shown hereafter, this simple model still provides useful qualitative information on the gyrotropic properties of asymmetric microcavities.

In the three following subsections the Kerr polarization rotation and phase change are studied quantitatively using Eqs. (3) to (6) and in the more qualitative way using Eq. (10) for different resonant optical nonlinearities.

\section{A. Saturation of the quantum well exciton}

For a $\hat{\sigma}_{+}$polarized excitation, $\Gamma_{0_{-}}=\Gamma_{0}$ and $\Gamma_{0_{+}}=\Gamma_{0}(1$ $-2 S_{+}$), where $S_{+}$represents the fraction of the upper-level population for the $\hat{\sigma}_{+}$polarized transition. Figures 4(a) and 4(b) show the photoinduced Kerr rotation and phase change spectra, respectively, plotted for different fraction of saturation of the $\hat{\sigma}_{+}$polarized transition $\left(S_{+}=0.015,0.03,0.045\right.$, and 0.06 in the continuous, dashed, dotted, and dash-dotted lines, respectively). All the rotation spectra shown in Fig. 4(a) exhibit the same "absorption-type" shape with positive and negative peaks located at the lower and upper polariton frequencies $\omega_{L}$ and $\omega_{U}$, respectively. On the other hand, the photoinduced phase change spectra of Fig. 4(b) appear as "dispersion-type" spectra centered on the polariton frequen- cies. Moreover, for the small values of the saturation factor used for this calculation, $\theta$ and $\varphi$ are proportional to $S_{+}$, and the maximum rotations and phase changes are large, making the device potentially interesting for optical signal processing. It must also be stressed that the amplitudes of both Kerr rotation and phase change are larger around the lower polariton mode than around the upper one. This surprising behavior is in fact related to the dephasing $\Phi_{\mathrm{QW}}$ brought by the quantum well transmission [see Eq. 6(b)] which widens and narrows the upper and lower polariton modes, respectively.

The main features appearing in Figs. 4(a) and 4(b) can be easily understood using the qualitative analysis of Eq. (10). First, the saturation of the $\hat{\sigma}_{+}$polarized exciton transition produces a small increase and decrease of $\omega_{L_{+}}$and $\omega_{U_{+}}$by the quantity $\Delta \Omega_{+} \approx S_{+} V_{0}^{2} / \Omega$, while the lower and upper polariton frequencies remain unchanged for the unsaturated $\hat{\sigma}_{-}$ polarized exciton transition. As a consequence, the photoinduced Kerr polarization rotation and phase change exhibit "absorption" and "dispersion" shapes, respectively, with opposite signs around $\omega_{L}$ and $\omega_{U}$, as shown in Fig. 4. Secondly, due to the proportionality of $\theta_{L, U}$ and $\varphi_{L, U}$ with respect to $\Delta \omega_{L, U}$, the maximum values of the photoinduced Kerr rotation and phase changes increases proportionally to $S_{+}$as it can be seen in Fig. 4. Finally, the lower values of $\theta_{U}$ and $\varphi_{U}$ when compared to those obtained around $\omega_{L}$ are easily understood taking the modifications of the polariton linewidths by the quantum well dephasing into account $\left(\gamma_{L}<\gamma_{U}\right)$.

\section{B. Blueshift of the quantum well exciton}

For a $\hat{\sigma}_{+}$polarized excitation, a blueshift occurs for the $\hat{\sigma}_{+}$excitonic transition and the $\hat{\sigma}_{-}$one remains unchanged (if no spin relaxation occurs during the experiment). As shown in Fig. 5, this nonlinearity also produces large rotations and phase changes for the light reflected by the microcavity even for the small blue shifts considered in this calculations $\left(\delta \omega_{0_{+}} / \gamma_{0}=0.05,0.1, \quad 0.15\right.$, and 0.2 in the continuous, dashed, dotted, and dash-dotted lines, respectively). As shown in Figs. 4(a) and 4(b), the same general features are observed for the photoinduced polarization and phase change spectra as in the case of the saturation of the $\hat{\sigma}_{+}$polarized transition. The main difference consists in the respective signs of $\theta$ and $\varphi$ around the upper and lower polariton frequencies. Indeed, the photoinduced Kerr rotation and phase change shown in Figs. 4(a) and 4(b) exhibit contributions of the same sign around $\omega_{L}$ and $\omega_{U}$. This is well explained using the qualitative model of Eqs. 10(a) and 10(b), since the modification of the polariton frequencies by the pump induced blueshift of the exciton frequency are the same for both polariton transitions $\left(\Delta \omega_{L, U}=\delta \omega_{0_{+}} / 2\right)$. Moreover, as $\Delta \omega_{L, U}$ have almost the same values for the choice of parameters made in Figs. 4 and 5, the maximum amplitudes of $\theta_{L, U}$ and $\varphi_{L, U}$ are almost the same.

\section{Saturation and blueshift of the quantum well exciton}

Actually, in most semiconductor quantum wells, saturation and blueshift of the exciton transition occur together. ${ }^{21}$ It 


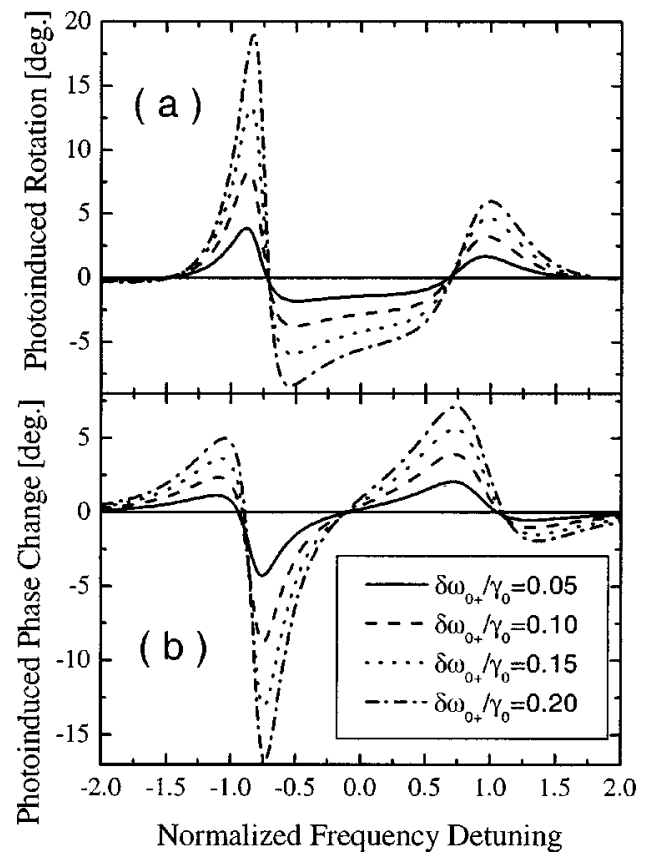

FIG. 5. Photoinduced Kerr rotation (a) and phase change (b) spectra for various blue shifts of the $\sigma_{+}$polarized exciton transition.

is, then, necessary to take their contributions to the photoinduced Kerr rotation and phase change simultaneously into account. The result of the quantitative model using the optical scattering matrices is presented in Figs. 6(a) and 6(b). In these figures the rotation and phase change spectra are obtained using the same parameters $S_{+}$and $\delta \omega_{0_{+}} / \gamma_{0}$ as in the case of Figs. 4 and 5, respectively. Spectra appearing in Fig.

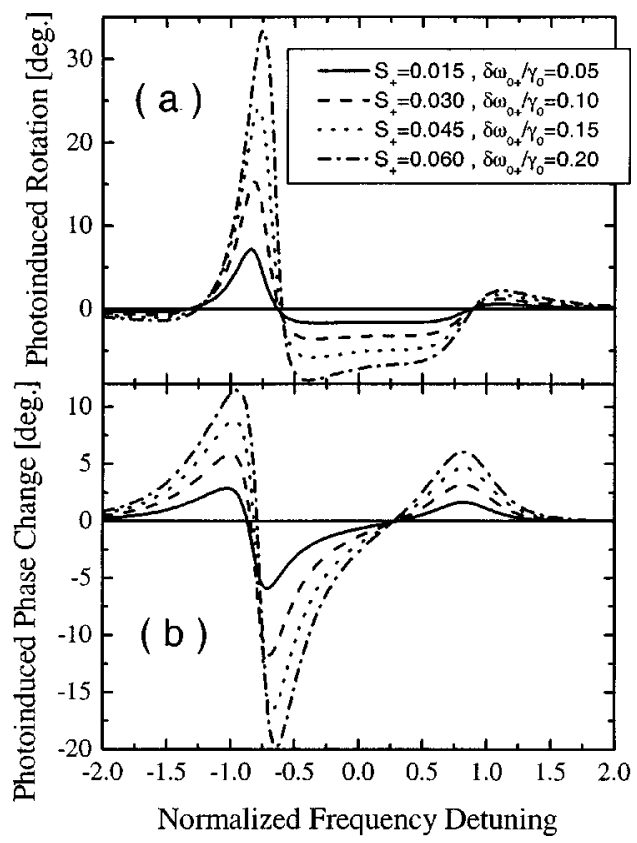

FIG. 6. Photoinduced Kerr rotation (a) and phase change (b) spectra for various saturations and blueshifts of the $\sigma_{+}$polarized exciton transition.
6 look similar to the algebraic sum of spectra shown in Figs. 4 and 5. They exhibit an enhancement and a quasisuppression of the photoinduced Kerr rotation and phase change around the lower and upper polariton frequencies, with a ratio of about 10 for the contributions occurring around these frequencies [this calculation corresponds to the fit to the experimental results shown in Fig. 3(a)]. This behavior is easily understood using the qualitative model since the lower polariton frequency is strongly blueshifted $\left(\Delta \omega_{L} \approx \delta \omega_{0_{+}} / 2\right.$ $\left.+S_{+} V_{0}^{2} / \Omega\right)$ while the upper one almost remains unchanged $\left(\Delta \omega_{L} \approx \delta \omega_{0_{+}} / 2-S_{+} V_{0}^{2} / \Omega \approx 0\right)$, with a ratio of about 6 for $\Delta \omega_{L} / \Delta \omega_{U}$ for the parameters used in our calculations. This value is in good agreement with the observations made from Fig. 6. Let us also remark that, unfortunately, saturation and blueshift of the quantum well exciton are both due to the photogeneration of high exciton densities. Thus, their contributions to the photoinduced Kerr effects cannot be separated, which enforces a two parameter fit of the experimental results.

\section{Photoinduced pseudo-Zeeman effect}

In the case of semimagnetic semiconductor quantum wells, relatively high magnetization can be photoinduced by circularly polarized pump light due to inverse exchange interaction. ${ }^{22}$ This magnetization is proportional to the density difference $n_{+}-n_{-}$of $\hat{\sigma}_{+}$and $\hat{\sigma}_{-}$polarized excitons photoinduced by the $\hat{\sigma}_{+}$polarized pump pulse. The amplitude of the magnetization results from the tradeoff between the exciton spin relaxation and the spin orientation of magnetic ions. At low temperatures, the exciton spin relaxation time $\tau_{S}$ is smaller than the spin orientation time $T_{M}$ of the magnetic ions, and the photoinduced magnetization is low and reaches its maximum after the short duration pump pulse. At high temperatures, $T_{M}$ is shorter, and the magnetization can be high during the pump pulse. In such a case, due to exchange interaction, ${ }^{24}$ giant Kerr rotations and phase changes of the polarization state of the incident probe pulse are possible. Figures 7(a) and 7(b) show Kerr rotation and phase change spectra, respectively, for several values of the pseudo-Zeeman splitting $\left(\delta \omega_{Z}=0.05,0.1,0.15\right.$, and 0.2 in the continuous, dashed, dotted, and dash-dotted lines, respectively). These spectra are nearly the opposite of those shown in Fig. 5 as confirmed by the qualitative analysis since lower and upper polariton modes are blueshifted by $-\delta \omega_{Z} / 2$ and $+\delta \omega_{Z} / 2$ for $\hat{\sigma}_{+}$and $\hat{\sigma}_{-}$polarizations, respectively.

\section{INFLUENCE OF EXCITON SPIN RELAXATION}

The results presented in Figs. 4-7 do not take exciton spin relaxation into account. However, this process can modify the values of the photoinduced Kerr rotations and phase changes in different ways. In this section, we consider two situations in the case when $T_{M}$ is much longer than $\tau_{S}$, so that almost no magnetization is created in the microcavity.

First, for pump and probe pulse duration $\Delta t$ shorter than $\tau_{S}$, the saturation and blueshift of the exciton transition are fully $\hat{\sigma}_{+}$polarized for pump-probe delays smaller than $\tau_{S}$. For larger delays, spin relaxation reduce the density differ- 


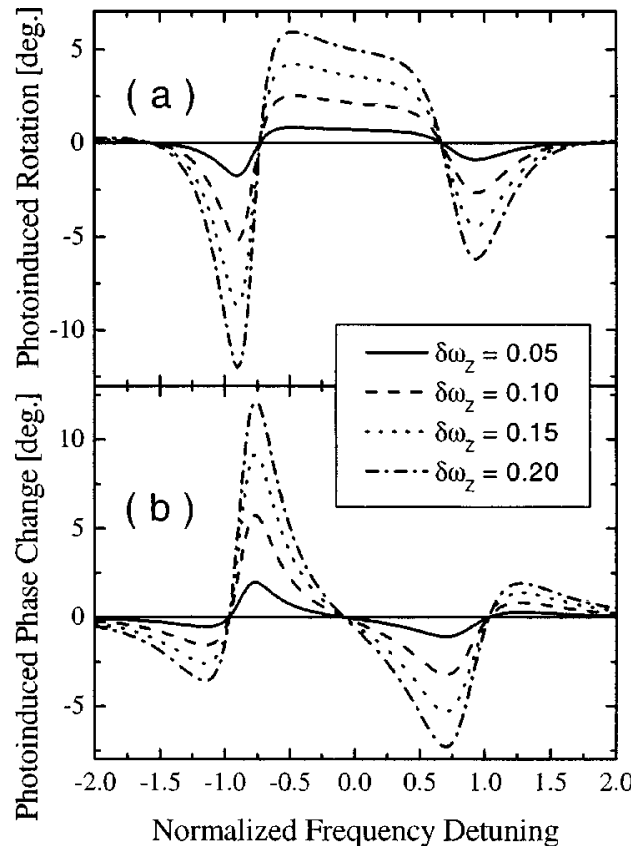

FIG. 7. Photoinduced Kerr rotation (a) and phase change (b) spectra for various $\sigma_{+}$polarized exciton induced Zeeman splittings.

ence $n_{+}-n_{-}$between $\hat{\sigma}_{+}$and $\hat{\sigma}_{-}$polarized excitons, while the sum $n_{+}+n_{-}$remains constant whenever the pump-probe delay is shorter than the exciton lifetime. Evidently, this reduction of the spin density difference modifies the Kerr rotation and phase change spectra since both $S_{ \pm}$and $\delta \omega_{0_{+}}$are proportional to the normalized $\hat{\sigma}_{ \pm}$density $n_{ \pm} /\left(n_{+}+n_{-}\right)$.

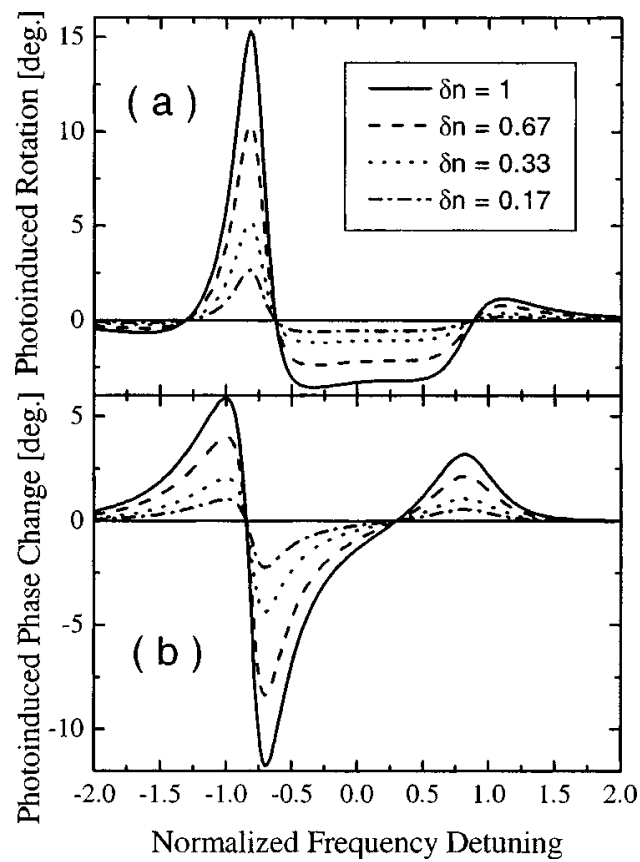

FIG. 8. Evolution of the photoinduced Kerr rotation (a) and phase change (b) spectra during the exciton spin relaxation. Spectra are represented from the initial excitation by $\sigma_{+}$polarized pump photons before the spin relaxation $(\delta n=1)$ to a nearly complete spin relaxation $(\delta n=0.17)$.

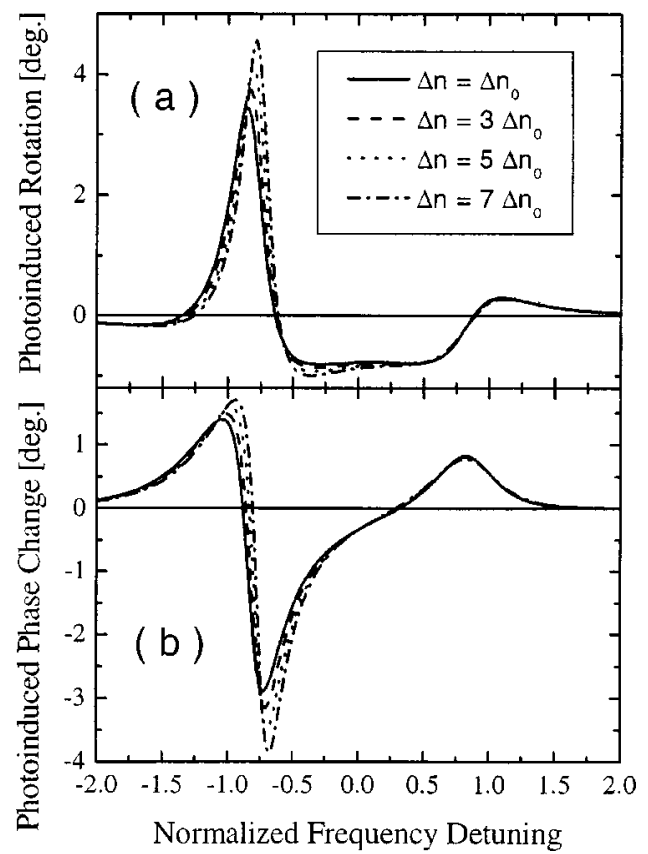

FIG. 9. Influence of spin relaxation occurring during the pump pulse duration on the photoinduced Kerr rotation (a) and phase change (b) spectra. Spectra are represented for different total exciton densities $\Delta n$, the spin density difference $n_{+}-n_{-}$being kept constant to $\Delta n_{0}$.

This is illustrated in Figs. 8(a) and 8(b) which show the Kerr rotation and phase change spectra, respectively, for several values of the normalized density difference $\delta n=\left(n_{+}\right.$ $\left.-n_{-}\right) /\left(n_{+}+n_{-}\right)(\delta n=1,0.67,0.33$, and 0.17 in the continuous, dashed, dotted, and dash-dotted lines, respectively). The spectra of Fig. 8 look exactly like those obtained in Fig. 6 in the absence of exciton spin relaxation. This indicates that for a given total exciton density the time delayed photoinduced Kerr rotation and phase change spectra can be deduced from those obtained initially $(\delta n=1)$ simply by multiplying the rotation and phase angles by $\delta n$. Let us note, however, that this simple rule also predicted by the qualitative analysis of Eq. 10 is no longer valid at larger total excitation yields when high exciton densities are photogenerated by the pump pulse.

We also considered the case of synchronized pump and probe pulses of duration $\Delta t$ longer than $\tau_{S}$. As a consequence of spin relaxation, $\hat{\sigma}_{+}$and $\hat{\sigma}_{-}$polarized excitons are simultaneously present even for a $\hat{\sigma}_{+}$polarized pump pulse. In such a case, higher pump excitations are required for getting the same Kerr rotation and phase change spectra. This is illustrated in Fig. 9 which shows such spectra calculated for different total excitations $\Delta n=n_{+}+n_{-}$, keeping the difference $n_{+}-n_{-}$constant $\left(\Delta n=\Delta n_{0}, 3 \Delta n_{0}, 5 \Delta n_{0}\right.$, and 7 $\Delta n_{0}$ in the continuous, dashed, dotted, and dash-dotted lines, respectively). Spectra of Fig. 9 are very similar to each other with a slight modification of only $25 \%$ of the maximum photoinduced Kerr rotation and phase change angles for a multiplication by 7 of the total excitation. This observation is in good agreement with the prediction of the qualitative analysis since $\Delta \omega_{L, U}$ only depends on the difference be- 
tween the $\hat{\sigma}_{+}$and $\hat{\sigma}_{-}$polarized exciton densities. This result definitely supports the gyrotropic nature of the photoinduced magneto-optic Kerr effects in semiconductor microcavities. It is also interesting concerning applications to optical signal processing since the repetition rate of operations has only to be smaller than the spin relaxation rate independently of the exciton lifetime.

\section{CONCLUSION}

Giant photoinduced Kerr effects have been predicted and measured in asymmetric semiconductor microcavities operated in the limit of the strong coupling regime. Before any relaxation processes, Kerr polarization rotation and ellipticity spectra calculated using the optical scattering matrices revealed positive or negative peaks around the polariton frequencies. These giant rotations and phase changes have been shown to occur due to the saturation, blueshift, and pseudoZeeman splitting of the exciton transition which strongly modified the coupling existing between the cavity and exciton modes. The intensity of the effect is, indeed, strongly amplified when compared to a bare single quantum well due to the sharp variation with frequency of the phase of the beam reflected by the microcavity around the polariton fre- quencies. A simple model involving a couple of two-level systems using the lower and upper polariton frequencies as the resonance frequencies has been shown to qualitatively describe all the features of the calculated spectra with the advantage of a simple physical interpretation of the results.

The role played by spin relaxation in the photoinduced Kerr rotation and phase change spectra have also confirmed the gyrotropic nature of the interaction with spectra almost insensitive to the total density of excitons and very dependent on the difference between the densities of counterrotating circularly polarized excitons.

A measure of photo-induced Kerr rotation performed at a temperature of $50 \mathrm{~K}$ in a microcavity containing a single semimagnetic semiconductor quantum well has confirmed the effectiveness of the effect with a polarization rotation of $10^{\circ}$ around the lower polariton frequency at a pump fluence of only $2 \mu \mathrm{J} / \mathrm{cm}^{2}$, in very good agreement with theory.

Due to the physical origin of the observed polarization rotation (reduction of the actual coupling of the cavity and exciton modes by the blue shift and saturation of the exciton transition), the process should persist at room temperature which could make such a device very promising for high contrast optical signal processing.
${ }^{1}$ C. Weissbuch, M. Nishioka, A. Ishikawa, and Y. Arakawa, Phys. Rev. Lett. 69, 3314 (1992).

${ }^{2}$ M. S. Skolnick, T. A. Fisher, and D. M. Whittaker, Semicond. Sci. Technol. 13, 645 (1998).

${ }^{3}$ T. A. Fisher, A. M. Afshar, D. M. Whittaker, J. S. Roberts, G. Hill, and M. A. Pate, Phys. Rev. B 51, 2600 (1995); Solid-State Electron. 40, 493 (1996).

${ }^{4}$ J. Tignon, P. Voisin, C. Delalande, M. Voos, R. Houdré, V. Oesterle, and R. P. Stanley, Phys. Rev. Lett. 74, 3967 (1995); Phys. Rev. B 56, 4068 (1997).

${ }^{5}$ T. A. Fisher, A. M. Afshar, M. S. Skolnick, D. M. Whittaker, and J. S. Roberts, Phys. Rev. B 53, R10469 (1996).

${ }^{6}$ A. Armittage, R. P. Stanley, M. S. Skolnick, D. M. Whittaker, P. Kinsler, and J. S. Roberts, Phys. Rev. B 55, 16395 (1997).

${ }^{7}$ J. D. Berger, O. Lyngues, H. M. Gibbs, G. Khitrova, T. R. Nelson, E. K. Lindmark, A. V. Kavokin, M. A. Kaliteevskii, and V. V. Zapasskii, Phys. Rev. B 54, 1975 (1996).

${ }^{8}$ M. Haddad, R. André, R. Frey, and C. Flytzanis, Solid State Commun. 111, 61 (1999).

${ }^{9}$ Le Si Dang, R. André, D. Heger, Y. Merle d'Aubigné, J. Cryst. Growth 184-185, 758 (1998); R. André, F. Boeuf, D. Heger, Le Si Dang, R. Romestain, J. Bleuse, and M. Müller, Acta Phys. Pol. 96, 511 (1999).

${ }^{10}$ R. Houdré, J. L. Gibernon, P. Pellandini, R. P. Stanley, U. Oesterle, C. Weisbuch, J. O'Gorman, B. Roycroft, and M. Ilegems, Phys. Rev. B 52, 7810 (1995).

${ }^{11}$ L. F. Stokes, M. Chodorow, and H. J. Shaw, Opt. Lett. 7,
288 (1982).

${ }^{12}$ F. Gires and P. Tournois, C. R. Acad. Sci. (Paris) 258, 6112 (1964).

${ }^{13}$ L. Menez, I. Zaquine, A. Maruani, and R. Frey, J. Opt. Soc. Am. B 16, 1849 (1999).

${ }^{14}$ M. Haddad, R. André, R. Frey, and C. Flytzanis, Solid State Commun. 111, 61 (1999).

${ }^{15}$ A. V. Kavokin, M. R. Vladimirova, M. A. Kaliteevski, O. Lyngnes, J. D. Berger, H. M. Gibbs, and G. Khitrova, Phys. Rev. B 56, 1087 (1997).

${ }^{16}$ J. Y. Huang, J. Y. Zhang, and Y. R. Shen, Appl. Phys. Lett. 57, 1961 (1990).

${ }^{17}$ R. Pankoke, A. Cassinelli, P. Sillard, C. Dorrer, P. Leisching, and R. Frey, Ann. Phys. (Paris) 20, 593 (1995).

${ }^{18}$ Y. Weissman, Optical Network Theory (Artec House, Norwood, MA, 1992).

${ }^{19}$ H. A. McLeod, Thin-Film Optical Filters, 2nd ed. (Hilger, London, 1986).

${ }^{20}$ E. L. Ivchenko, Sov. Phys. Solid State 33, 1344 (1991).

${ }^{21}$ S. Schmitt-Rink, D. S. Chemla, and D. A. B. Miller, Phys. Rev. B 32, 6601 (1985).

${ }^{22}$ H. Krenn, K. Kaltenegger, T. Dietl, J. Spalek, and G. Bauer, Phys. Rev. B 39, 10918 (1989).

${ }^{23}$ V. Savona, L. C. Andreani, P. Schwendimann, and A. Quattropani, Solid State Commun. 93, 733 (1995).

${ }^{24}$ J. A. Gaj, J. Ginter, and R. R. Galaska, Phys. Status Solidi B 89, 655 (1978). 